Published in final edited form as:

Semin Nephrol. 2017 November ; 37(6): 538-545. doi:10.1016/j.semnephrol.2017.07.007.

\title{
The cell biology of APOL1
}

\author{
John F. O’Toole, Leslie A. Bruggeman, Sethu Madhavan, and John R. Sedor ${ }^{\star}$
}

Department of Medicine, Case Western Reserve University and the Division of Nephrology, MetroHealth System, Cleveland, OH "Department of Physiology and Biophysics, Case Western Reserve University and the Division of Nephrology, MetroHealth System, Cleveland, $\mathrm{OH}$

\section{Abstract}

The association of variants in the APOL1 gene, which encodes apolipoprotein L1 (APOL1), with progressive non-diabetic kidney diseases in African Americans has prompted intense investigation into the function(s) of APOL1. APOL1 is an innate immune effector that protects humans from infection by some trypanosomal parasites. We review the data characterizing APOL1 trypanolytic function, which has been a basis for studies of APOL1 function in mammalian cells. Subsequently, we discuss the studies that use animal models, mammalian cell culture models, and kidney biopsy tissue to discover the mechanisms of variant APOL1-associated kidney diseases.

\section{Introduction}

The landmark discovery that genetic variations in the $A P O L 1$ gene associate with nondiabetic kidney diseases in individuals with African ancestry ${ }^{1,2}$ has prompted investigation into biologic mechanisms underlying the association. $A P O L 1$ encodes the apolipoprotein $\mathrm{L} 1$ protein (APOL1), which primarily circulates bound to HDL particles. This circulating fraction of APOL1 imparts resistance to trypanosomiasis by rapidly lysing infecting trypanosomes of the Trypanosoma brucei species $^{3}$, trypanosome parasites endemic to Africa. However, several T. brucei subspecies, T. b. rhodesiense and T. b. gambiense, which cause African Sleeping Sickness, developed mechanisms to resist APOL1-dependent killing. The APOL1 kidney disease-associated polymorphisms encode APOL1 proteins that circumvent immune evasion by the parasite and restore parasite killing to the resistant subspecies, but if present on both alleles, strongly increase chronic kidney disease (CKD) risk. The $A P O L 1$ gene (and trypanolytic activity) is only present in humans and several other primate species ${ }^{4-6}$, which complicates design of in vitro and animal models to explore the pathobiology of variant APOL1-associated kidney diseases. In this review article, we focus on data primarily from experimental models, which describe potential mechanisms of APOL1-associated kidney injury. Human kidney biopsy gene expression data addressing APOL1 function are also reviewed. In the following discussion, APOL1-G0 indicates the

Publisher's Disclaimer: This is a PDF file of an unedited manuscript that has been accepted for publication. As a service to our customers we are providing this early version of the manuscript. The manuscript will undergo copyediting, typesetting, and review of the resulting proof before it is published in its final citable form. Please note that during the production process errors may be discovered which could affect the content, and all legal disclaimers that apply to the journal pertain. 
"wild-type" or reference sequence; APOL1-G1 and APOL1-G2 are the kidney disease associated proteins.

\section{APOL1 and trypanolysis}

The mechanisms of APOL1's trypanosome-killing activity and the trypanosome's response have been the prime drivers of hypotheses about the pathways mediating APOL1-associated kidney injury (Figure 1). Humans and some higher primates resist trypanosomiasis by $T$. brucei, an arthropod-borne multispecies pathogen that limits agrarian activity in sub-Saharan Africa because livestock are primary hosts. Two trypanosome lytic factors (TLF), constitutively circulating as innate immune effectors, mediate this protection, and both contain the primate-only proteins, APOL1 and haptoglobin-related protein (HPR). Interestingly, genetic variants in the HPR locus associate with circulating APOL1 levels ${ }^{7}$, suggesting the biogenesis of the component proteins comprising TLFs is coordinated.

APOL1 is the trypanolytic molecule within both TLFs, and APOL-G0 protects humans from trypanosomiasis by the ancestral T. b. brucei. Only TLF-1 will be further discussed; more data about both TLFs are summarized in an excellent, recent review ${ }^{8}$. TLF-1 particles are a subclass of high density lipoprotein particles (HDL), which are lipid-rich and contain apolipoprotein A1 in addition to APOL1 and HPR. To evade the human innate immune response conferred by the TLFs, $T$. $b$. rhodesiense and T. . gambiense, have adapted by antigenic variation in one of their variant surface glycoproteins (VSG) expressed on the trypanosomal plasma membrane. Trypanosome VSGs are encoded from over 1000 transcription units within the trypanosome genome in 15 expression sites. Transcriptional switching between expression sites and/or by homologous recombination of VSG genes ${ }^{8}$ allows the parasite to elude host humoral immune defenses and other environmental stresses. A VSG, serum response-associated (SRA) protein, is expressed by one of the human disease-causing trypanosomes (T.b. rhodesiense) and binds APOL1-G0, blocking its trypanolytic activity. To counter this parasite adaption, $A P O L 1$ sequence variants, encoding APOL1-G1 and APOL-G2, evolved by positive selection ${ }^{1}$. Both variant APOL1 proteins have low affinity for SRA and restore parasite killing and resistance to trypanosomiasis, albeit at increased susceptibility to chronic kidney diseases in humans with two risk alleles.

For trypanolysis to occur, TLF-1 is endocytosed into the parasite by a specific receptor for haptoglobin-hemoglobin, which supplies heme iron necessary for growth and resistance to host's oxidative response ${ }^{9}$. HPR is the ligand for the trypanosomal receptor, facilitating the uptake of TLF-1. A number of studies have demonstrated that TLF-1 traffics through the endolysosomal pathway, ultimately accumulating in lysosomes ${ }^{3,10-12}$. However, other evidence has suggested TLF-1 or APOL1 also targets, at least in part, to plasma membrane or the mitochondria, respectively ${ }^{13-15}$. Early studies suggested SRA bound TLF-1 in the lysosome to block its toxicity and protect the parasite ${ }^{11}$. More recent data have shown SRA and TLF-1 may actually interact in early endosomes and that SRA accelerates TLF-1 degradation ${ }^{16}$.

The mechanism of trypanosomal cell death due to TLF-1 remains unclear. APOL1 is the trypanolytic toxin ${ }^{8}$ in both TLFs, although some studies suggest both HPR and APOA1 are also necessary ${ }^{12,17}$. The APOL1 aa 60 - aa235 region has marginal similarity to the pore- 
forming domains of the bacterial colicins, which are bactericidal toxins. In an acidic environment found in some endosomes and lysosomes, APOL1 undergoes conformational changes that facilitates its insertion into membranes. The initial report showed lysosomal membrane targeting resulting in chloride influx ${ }^{11}$. However others demonstrated APOL1 targets the plasma membrane through endosomal recycling and functions as a non-selective, cation channel ${ }^{13,14,18}$. In contrast to data that have demonstrated low $\mathrm{pH}$ allows ion fluxes, other investigators have found that APOL1, inserted into membranes in acid environments, remains inactive, only permitting ion flux when exposed to physiological $\mathrm{pH}^{18}$. Lysosomal swelling may be the proximate cause of trypanosomal death ${ }^{11}$. Other data suggest sodium influx through APOL1 pores in the plasma membrane generates osmotic stress with cytoplasmic swelling and trypanolysis ${ }^{13,14,18}$ APOL1-depedent osmotic lysis can be blocked by the anti-oxidant DPPD, suggesting APOL1-dependent oxidative stress also results from its pore-forming activity ${ }^{14}$. Trypanolysis has been dissociated from lysosomal swelling ${ }^{15}$, suggesting the lysosomal localization of APOL1 may reflect the lysosome's role as a detoxifying pathway for the parasite rather than key effector of parasite lysis. Rather, some APOL1-containing endosomes escape from the endolysosomal pathway and are trafficked by a trypanosomal kinesin to the mitochondria. Here APOL1 causes mitochondrial membrane permeabilization permitting release of a trypanosomal endonuclease and resulting in DNA fragmentation and trypanolysis.

In aggregate, the data strongly support that APOL1 is the trypanolytic toxin and that it undergoes a conformational change in an acid environment to insert into a lipid membrane. APOL1-G0, -G1 and -G2 have pore-forming or channel like activity in trypanosomes; however, data conflict on the membrane target, channel selectivity, and effect of acid $\mathrm{pH}$ on ion flux. Channel activity has been characterized as an acid-activated chloride channel and as an acid-gated cation channel. The specific mechanism of trypanolysis remains unclear; lysosomal membrane disruption, lysosomal swelling, osmotic lysis and apoptosis-like cell death are all mechanisms proposed to cause parasite death. Trypanolytic mechanisms have informed the design of many of the experiments discussed below, which focus on pathways of variant APOL1-dependent kidney injury.

\section{APOL1 tissue and cellular distribution}

APOL1 is a circulating trypanolytic toxin, and intriguingly, circulating factors may cause focal segmental glomerulosclerosis (FSGS), a kidney disease associated with APOL $1 \mathrm{G} 1$ and $\mathrm{G} 2$ variants ${ }^{19}, 20$. However, the $A P O L 1$ gene is also expressed in many tissues including the kidney $6,21,22$. This broad tissue distribution suggests that, in addition to its role as a circulating trypanolytic innate immune effector, APOL1 may have a local role in the tissues where it is expressed, either para- or intracellularly. Therefore either circulating variant APOL1 and/or kidney-associated APOL1 could increase the risk for kidney diseases associated with the $A P O L 1 \mathrm{G} 1$ or $\mathrm{G} 2$ variants. Published evidence provides strong support for the premise that kidney-associated APOL1-G1 and/or -G2 mediate disease. Circulating total APOL1 and HDL levels failed to correlate with kidney disease phenotypes in African American HIV subjects followed in the ALLRT cohort ${ }^{23}$ or in the Dallas Heart Study ${ }^{7}$. In the latter study, APOL1-G0, -G1 and G2 were quantified by LC-MS. An additional analysis of allele-specific APOL1 with kidney phenotypes, restricted to individuals who carried at 
least one copy of the corresponding allele, still failed to show an association with chronic kidney disease, eGFR or albuminuria. In a second approach to assess the contributions of circulating and kidney-associated variant APOL1 to disease, the APOL1 genotypes of transplanted kidneys and/or the kidney transplant recipients have been ascertained in several cohorts ${ }^{24-26}$. Risk for graft loss associated with the genotype of the transplanted organ, but not with the genotype of the recipient.

In normal human kidney the APOL1 protein localizes to the podocyte, the proximal tubule and the endothelium of extraglomerular arterioles and small arteries ${ }^{27}$, a finding subsequently replicated by others ${ }^{28,} 29$. Interestingly, the amount of APOL1 protein in the podocyte and proximal tubules was decreased in FSGS or HIV associated nephropathy (HIVAN), two kidney diseases associated with APOL1 risk variants ${ }^{27,}{ }^{29}$. Although, the APOL1 signal in the vascular endothelium appeared similar in disease and non-disease kidney sections, the vascular media of renal arterioles and small arteries stained positive only in the setting of kidney disease ${ }^{27}$. Localization of APOL1 to the podocyte is consistent with its central role in the pathogenesis of many of the kidney diseases associated with APOL1. However, the localization of APOL1 to renal cells outside of the glomerulus raises the possibility that these cell types and a functional role for APOL1 within them may contribute to the phenotypes of some renal pathologies such as the proximal tubule in HIVAN or the vascular media and endothelium in hypertension associated kidney disease. The functional role of APOL1 within any of these cell types remains unknown.

\section{APOL 1 abundance and kidney disease}

APOL1 expression is induced by TNF-a, an inflammatory cytokine, in cultured endothelial cells $^{22}$ and cancer cells ${ }^{30}$. Cytokine induction of $A P O L 1$ expression was subsequently confirmed in cultured kidney microvascular endothelial cells and podocytes ${ }^{27,31,32}$. The APOL1 promoter contains regulatory elements for the transcription factors STAT2, interferon regulating factor (IRF) 1 and IRF2 ${ }^{32}$, suggesting that $A P O L 1$ is a cellular immune response gene, whose expression is stimulated by viral infection. This speculation is supported by experiments demonstrating increased expression of $A P O L 1$ after treatment of cultured cells with poly I:C, which mimics double stranded RNA and models viral infection $^{32}$. Together these data suggest that APOL1 is part of an anti-viral response, an idea consistent with the strong association of the $A P O L 1 \mathrm{G} 1$ and $\mathrm{G} 2$ variants with HIVAN ${ }^{33}$. APOL1-G0 may prevent uncontrolled HIV infection of the kidney or the APOL1-G1 andG2 variants in the setting of HIV infection may mediate kidney injury and fail to stop HIV infection.

Although inflammatory mediators consistently increase APOL1 expression in vitro, immunohistochemical studies of human kidney sections of individuals with kidney diseases demonstrate decreased abundance of APOL1 protein in the podocyte and proximal tubule $^{27,29}$. Subjects with kidney diseases and the APOL1 risk genotype [two risk alleles] have similar APOL1 abundance by immunohistology compared to subjects with kidney disease and the low risk genotype [one on no risk alleles]. Another study compared APOL1 transcript levels in glomerular and tubulointerstitial compartments microdissected from human kidney biopsy samples of nephrotic syndrome subjects. Concordant with the 
immunohistology, APOL1 mRNA in both compartments was similar between nephrotic syndrome subjects with and without $A P O L 1$ risk genotypes ${ }^{34}$. These data suggest that APOL1-associated kidney diseases do not result from differential abundance of variant APOL1 compared to APOL1-G0. Of course, both studies used clinically indicated biopsies, reflecting study populations with more advanced kidney diseases that may have obscured variant-dependent differences in APOL1 abundance earlier in the disease course. APOL1 levels have not been compared between kidney diseases associated with APOL1 variants (e.g. HIVAN and FSGS) and those that are not (e.g. diabetic nephropathy and IgA nephropathy). Further study will be needed to reconcile discrepant assessment of APOL1 abundance between the cell culture models and kidney biopsy observations, and determine if APOL1 transcription or translation is dysregulated in kidney disease.

\section{APOL1-associated cytotoxicity}

Similar to its function as a trypanosome toxin, ectopic expression of both APOL1-G0 and the variant APOL1s in mammalian cells can cause cytotoxicity ${ }^{31,32,35-38}$. As previously mentioned, the APOL1 protein has homology to the bacterial pore-forming toxins of the colicin family ${ }^{11}$, which have channel-like properties and APOL1-mediated cytotoxicity is dependent upon channel activity ${ }^{38,} 39$. In these reports the expression of APOL1-G0 or its kidney disease-associated variants are associated with cytotoxicity in stable expression in cell culture ${ }^{38}$ or when expressed in Xenopus oocytes ${ }^{39}$. APOL1 -G1 and -G2 in cultured cells caused increased cytotoxicity and cell swelling compared to cells expressing APOL1G0. In contrast, expression of APOL1 in the Xenopus oocyte model failed to detect a difference in cytotoxicity between the G0 and the G1 or G2 risk variants. An important piece of data regarding APOL1-mediated cytotoxicity that will need to be addressed in future studies is whether variant-dependent cytotoxicity also occurs in model systems where APOL1 expression is under control of its endogenous promoter and ultimately in APOL1associated kidney diseases.

Cytotoxicity in extra-renal cells and tissues has also been observed in two published reports. The first examines the stable expression of APOL1-G0, -G1, and -G2 in hepatocytes ${ }^{36}$. APOL1-G0,-G1 and-G2 expression caused cytotoxicity when stably expressed in rat hepatoma cells without a consistent increase in risk variant cytotoxicity ${ }^{36}$. The second report utilized hydrodynamic gene delivery (HGD), a technique that allows the expression of a gene of interest in mice resulting in primarily hepatic expression. HGD was used to express the APOL1-G0, -G1, and -G2 variants in the liver and circulation of mice ${ }^{40}$. Although the APOL1 variants have not been associated with liver disease or damage, the authors observed severe hepatic necrosis after injection of constructs for the expression of APOL1-G1, which was less severe for $-\mathrm{G} 2$ and not observed with $-\mathrm{G} 0$. No renal phenotype was observed in these animals; however, the level of kidney expression of the transgene is much lower than hepatic expression when using the HGD technique.

Two papers have reported kidney phenotypes in model organisms. Morpholino or CRISPR/ Cas9 knockdown of zebrafish (zf) apoll caused pericardial edema, depressed glomerular filtration and podocyte foot process effacement ${ }^{29}, 41$, which was complemented by human APOL $1 \mathrm{G} 0$ but not $\mathrm{G} 1$ or $\mathrm{G} 2{ }^{41}$, suggesting variant APOL1 have lost an activity necessary 
for normal function of the pronephric glomerular filtration barrier. zfApol1 is expressed in podocytes and endothelial cells in the zebrafish pronephros and has $38 \%$ identity to human APOL $1{ }^{41,42}$. The rapid gene duplication of the human APOL1 gene cluster makes it difficult to determine homologues outside primate species. However, a case report of an individual with truncating mutations in APOL1 and normal kidney function ${ }^{43}$ suggests that the function of APOL1 may not be constitutive, but required in response to specific environmental challenges. We created transgenic ( $\mathrm{Tg}$ ) mice using the $\mathrm{Nphs} 1$ promoter to express APOL1-G0 or APOL1-G2 in podocytes ${ }^{44}$. Tg-G0 and Tg-G2 mice did not develop kidney pathology, proteinuria, or azotemia as of 300 days of age, and we could not demonstrate necrotic, apoptotic or autophagic cell death. However, podocyte density was significantly decreased by 200 days of age in Tg-G2 mice compared to age-matched wildtype and Tg-G0 mice. These data suggest APOL1 kidney disease risk variants may compromise the ability of the podocyte to maintain attachment during the hypertrophy of the glomerulus that occurs during normal aging ${ }^{45}$, 46 . In the absence of an additional environmental stressor, the degree of podocyte loss related to the APOL1 risk variants is clinically silent as was observed in the transgenic mouse model (Figure 2) but a "second hit," like HIV infection, could accelerate podocyte depletion and cause overt kidney disease.

\section{APOL1-activated signaling pathways}

Transient and stable expression of APOL1 in cell culture models has been used to study its function. An early report characterized APOL1-G0 as a BH3 domain containing protein and stable expression of APOL1-G0 in cancer cell lines resulted in cell death attributed to autophagy ${ }^{35}$. These authors found that mutation of the BH3 domain of APOL1-G0 abolished the cell death phenotype. A subsequent report found that this was not related to any functional role of the $\mathrm{BH} 3$ domain, but more likely related to altered protein structure resulting from deletion of nine amino acids comprising the $\mathrm{BH} 3$ domain $^{39}$. The role of autophagy in APOL1-mediated cell death has subsequently been examined in a number of reports, which have come to conflicting conclusions ${ }^{31,36-39}$. The transient expression APOL1-G0 in HEK293 cells led to an autophagic block due to failure of autophagosomeslysosome fusion but not autophagy induction and the use of inhibitors of autophagy did not block cell death ${ }^{37}$. Two studies examined cultured cells stably expressing APOL1 in podocytes $^{31}$ or HEK293 cells ${ }^{38}$. Both observed variant-dependent differences in APOL1mediated cell death. Lan et al did not directly measure autophagic flux, but treatment of cells expressing APOL1 with chloroquine, an inhibitor of lysosome acidification, a necessary step in autophagic maturation, attenuated APOL1 variant-dependent cell death ${ }^{31}$. Olabisi et al reported an increase in LC3II/LC3I, a biochemical index of autophagic flux, in cells expressing APOL1-G1, but not in cells expressing APOL1-G0 ${ }^{38}$. However, autophagy inhibitors did not block APOL1-mediated cytotoxicity. APOL1-G0, -G1, and-G2 all increased LC3II and caused cytotoxicity in rat hepatoma cells, which was reduced by blocking autophagy induction with 3-methyladenine, a class III phosphoinositide 3-kinase inhibitor ${ }^{36}$. Finally, in Xenopus oocytes expressing APOL1-G0,-G1 and-G2 caused morphologic toxicity ${ }^{39}$. Although autophagy was not directly assessed, treatment of oocytes with inhibitors of autophagy induction or maturation did not alter morphological deterioration. 
Reconciling these conflicting data is difficult given the diversity of models and experimental designs. Taken together, reference and variant APOL1 can induce autophagy in certain systems. However none of the published data allows a distinction between cause and effect. That is, does APOL1 directly stimulate autophagic pathways or does APOL1-mediated cell death trigger secondary activation of autophagic pathways? APOL1-dependent cytotoxicity results from ion fluxes after its insertion into the plasma membrane ${ }^{38,39}$, similar to mechanisms of trypanolysis reported by some but not all investigators ${ }^{13}, 14$. Pore forming toxins of pathogens can activate autophagy in cells $\mathrm{s}^{47,48}$ suggesting that the observed induction of autophagy by APOL1 expression systems may be a cellular response to APOL1 mediated channel activity. Finally, stable expression of APOL1 in cells may poorly model its activities in vivo. The APOL1 transgenic mice, described in the previous section, were crossed with another transgenic mouse model ubiquitously expressing a green fluorescent protein-tagged microtubule-associated protein 1 light chain 3 (GFP-LC3) ${ }^{44}$. LC3 labels autophagosomal membranes and GFP-LC3 can be visualized as fluorescent intracellular puncta. Autophagosome numbers in podocytes of wild type, Tg-G0 and Tg-G2 mice were equivalent, suggesting APOL1 expression alone is not sufficient to activate autophagy in healthy kidneys.

The channel activities of APOL1-G0 and variant APOL1s in mammalian cells have been characterized in several papers, again with conflicting results. APOL1 clearly has poreforming or channel functions when reconstituted in lipid bilayers, which has been variably demonstrated to be a selective chloride channel ${ }^{11}$ or a non-specific, cation channel that permitted $\mathrm{K}^{+}$efflux and $\mathrm{Na}^{+}$influx ${ }^{13,18}$. In cultured cells, APOL1-G2, but not APOL1-G0, inserted in the plasma membrane leading to loss of intracellular potassium ${ }^{38}$. p38 mitogen activated protein kinase (p38-MAPK) is activated by intracellular potassium loss after exposure of cells to pore-forming toxins ${ }^{49}$. Similarly, p-38-MAPK was activated in cells expressing APOL1-G1 or APOL1-G2 but not APOL1-G0. Activation of p38 MAPK is associated with kidney diseases ${ }^{50}$. In addition, both APOL1 variants downregulated gp130STAT signaling pathways, but the role of this pathway in APOL-1-dependent cell death and APOL1-associated kidney diseases is unclear. Some papers suggest STAT3 promotes podocyte differentiation. In contrast, increased STAT3 activity caused podocyte proliferation and dedifferentiation in FSGS and HIVAN ${ }^{51-53}$, kidney diseases strongly associated with APOL1 variants ${ }^{33,54}$.

HIV-1 infection of renal cells is required for HIVAN pathogenesis ${ }^{55}$, 56, suggesting APOL1G0 but not variant APOL1s could block HIV infection in kidney cells. Two papers suggest APOL1-G0 is a HIV restriction factor ${ }^{57,58}$, but neither paper assessed differences between APOL1-G0 and APOL1-G1 or -G2 in capability to restrict HIV infection. Taylor et al further explored the mechanism of APOL1-G0 as an HIV restriction factor and found APOL1-G0 caused nuclear translocation of transcription factor EB (TFEB) with subsequent increases in endocytosis and lysosomal biogenesis, which was concordant with a reduction of viral transcription and endolysosomal clearance of the viral proteins Vif and the endocytosis and Gag. Interestingly, a subsequent report found that, while necessary for APOL1 viral restriction function, TFEB nuclear translocation is not required for APOL1mediated cytotoxicity ${ }^{37}$. 
Currently little data addresses APOL1-G0,-G1 or -G2 function in human tissues where it is expressed under its endogenous promoter. As previously mentioned, APOL1 transcript levels were similar in glomerular or tubulointerstitial compartments of kidney biopsies from proteinuric African Americans with high and low risk APOL1 risk genotypes ${ }^{34}$. In addition, differential expression analysis, comparing transcriptomes from kidney biopsies of subjects with $A P O L 1$ high risk and low risk genotypes, identified only five differentially expressed genes (CXCL9, CXCL11, and UBD in glomerulus; SNOR14B and MUC13 in tubulointerstitium). Finally, these investigators used coregulation analysis to identified gene sets whose expression correlated with $A P O L 1$ transcript levels in the tubulointerstitial compartments of subjects with high risk versus low risk $A P O L 1$ genotypes. Pathway analysis of gene sets, whose expression correlated with APOL1 transcripts, suggested immune and inflammatory pathways are differentially activated in kidneys of proteinuric subjects with high risk $A P O L 1$ genotypes.

\section{Conclusions}

More than 20 million Americans have CKD and more than 500,000 patients have end-stage renal disease (ESRD). Health care for ESRD patients, who comprise less than $1 \%$ of Medicare recipients, accounts for over 7\% of Medicare expenditures, approximately $\$ 35$ billion annually. ESRD patients are at risk for accelerated cardiovascular disease and early death. African American CKD patients in the United States are four times more likely than white patients to progress to ESRD, and a single genetic susceptibility locus ( $A P O L 1$ ) explains much of this increased risk. In order to improve our prognostic, diagnostic and therapeutic approaches, we need to gain a better understanding of the biologic mechanisms underlying the genetic association of APOL1 variations and kidney disease.

\section{Acknowledgments}

This authors are supported by NIH grants DK097836 and DK 108329 and the Clinical and Translational Science Collaborative of Cleveland supported by the National Center for Advancing Translational Sciences grant UL1TR000439. SMM was supported by training grant DK007470.

\section{Literature cited}

1. Genovese G, Friedman DJ, Ross MD, et al. Association of trypanolytic ApoL1 variants with kidney disease in African Americans. Science. 2010; 329:841-5. [PubMed: 20647424]

2. Tzur S, Rosset S, Shemer R, et al. Missense mutations in the APOL1 gene are highly associated with end stage kidney disease risk previously attributed to the MYH9 gene. Hum Genet. 2010; 128:345-50. [PubMed: 20635188]

3. Vanhamme L, Paturiaux-Hanocq F, Poelvoorde P, et al. Apolipoprotein L-I is the trypanosome lytic factor of human serum. Nature. 2003; 422:83-7. [PubMed: 12621437]

4. Smith EE, Malik HS. The apolipoprotein L family of programmed cell death and immunity genes rapidly evolved in primates at discrete sites of host-pathogen interactions. Genome Res. 2009; 19:850-8. [PubMed: 19299565]

5. Lugli EB, Pouliot M, Portela Mdel P, et al. Characterization of primate trypanosome lytic factors. Mol Biochem Parasitol. 2004; 138:9-20. [PubMed: 15500911]

6. Page NM, Butlin DJ, Lomthaisong K, et al. The human apolipoprotein L gene cluster: identification, classification, and sites of distribution. Genomics. 2001; 74:71-8. [PubMed: 11374903]

7. Kozlitina J, Zhou H, Brown PN, et al. Plasma Levels of Risk-Variant APOL1 Do Not Associate with Renal Disease in a Population-Based Cohort. J Am Soc Nephrol. 2016 
8. Pays E, Vanhollebeke B, Uzureau P, et al. The molecular arms race between African trypanosomes and humans. Nat Rev Microbiol. 2014; 12:575-84. [PubMed: 24975321]

9. Vanhollebeke B, De Muylder G, Nielsen MJ, et al. A haptoglobin-hemoglobin receptor conveys innate immunity to Trypanosoma brucei in humans. Science. 2008; 320:677-81. [PubMed: 18451305]

10. Hager KM, Pierce MA, Moore DR, et al. Endocytosis of a cytotoxic human high density lipoprotein results in disruption of acidic intracellular vesicles and subsequent killing of African trypanosomes. J Cell Biol. 1994; 126:155-67. [PubMed: 8027174]

11. Perez-Morga D, Vanhollebeke B, Paturiaux-Hanocq F, et al. Apolipoprotein L-I promotes trypanosome lysis by forming pores in lysosomal membranes. Science. 2005; 309:469-72. [PubMed: 16020735]

12. Harrington JM, Howell S, Hajduk SL. Membrane permeabilization by trypanosome lytic factor, a cytolytic human high density lipoprotein. J Biol Chem. 2009; 284:13505-12. [PubMed: 19324878]

13. Molina-Portela Mdel P, Lugli EB, Recio-Pinto E, et al. Trypanosome lytic factor, a subclass of high-density lipoprotein, forms cation-selective pores in membranes. Mol Biochem Parasitol. 2005; 144:218-26. [PubMed: 16202458]

14. Greene AS, Hajduk SL. Trypanosome Lytic Factor-1 Initiates Oxidation-stimulated Osmotic Lysis of Trypanosoma brucei brucei. J Biol Chem. 2016; 291:3063-75. [PubMed: 26645690]

15. Vanwalleghem G, Fontaine F, Lecordier L, et al. Coupling of lysosomal and mitochondrial membrane permeabilization in trypanolysis by APOL1. Nat Commun. 2015; 6:8078. [PubMed: 26307671]

16. Stephens NA, Hajduk SL. Endosomal localization of the serum resistance-associated protein in African trypanosomes confers human infectivity. Eukaryot Cell. 2011; 10:1023-33. [PubMed: 21705681]

17. Shiflett AM, Bishop JR, Pahwa A, et al. Human high density lipoproteins are platforms for the assembly of multi-component innate immune complexes. J Biol Chem. 2005; 280:32578-85. [PubMed: 16046400]

18. Thomson R, Finkelstein A. Human trypanolytic factor APOL1 forms pH-gated cation-selective channels in planar lipid bilayers: Relevance to trypanosome lysis. Proc Natl Acad Sci U S A. 2015; 112:2894-9. [PubMed: 25730870]

19. Savin VJ, Sharma R, Sharma M, et al. Circulating factor associated with increased glomerular permeability to albumin in recurrent focal segmental glomerulosclerosis. N Engl J Med. 1996; 334:878-83. [PubMed: 8596570]

20. Wei C, El Hindi S, Li J, et al. Circulating urokinase receptor as a cause of focal segmental glomerulosclerosis. Nat Med. 2011; 17:952-60. [PubMed: 21804539]

21. Duchateau PN, Pullinger CR, Cho MH, et al. Apolipoprotein L gene family: tissue-specific expression, splicing, promoter regions; discovery of a new gene. J Lipid Res. 2001; 42:620-30. [PubMed: 11290834]

22. Monajemi H, Fontijn RD, Pannekoek H, et al. The apolipoprotein L gene cluster has emerged recently in evolution and is expressed in human vascular tissue. Genomics. 2002; 79:539-46. [PubMed: 11944986]

23. Bruggeman LA, O’Toole JF, Ross MD, et al. Plasma apolipoprotein L1 levels do not correlate with CKD. J Am Soc Nephrol. 2014; 25:634-44. [PubMed: 24231663]

24. Reeves-Daniel AM, DePalma JA, Bleyer AJ, et al. The APOL1 gene and allograft survival after kidney transplantation. Am J Transplant. 2011; 11:1025-30. [PubMed: 21486385]

25. Lee BT, Kumar V, Williams TA, et al. The APOL1 genotype of African American kidney transplant recipients does not impact 5-year allograft survival. Am J Transplant. 2012; 12:1924-8. [PubMed: 22487534]

26. Freedman BI, Pastan SO, Israni AK, et al. APOL1 Genotype and Kidney Transplantation Outcomes From Deceased African American Donors. Transplantation. 2016; 100:194-202. [PubMed: 26566060]

27. Madhavan SM, O'Toole JF, Konieczkowski M, et al. APOL1 localization in normal kidney and nondiabetic kidney disease. J Am Soc Nephrol. 2011; 22:2119-28. [PubMed: 21997392] 
28. Ma L, Shelness GS, Snipes JA. Localization of APOL1 Protein and mRNA in the Human Kidney: Nondiseased Tissue, Primary Cells, and Immortalized Cell Lines. J Am Soc Nephrol. 2014

29. Kotb AM, Simon O, Blumenthal A, et al. Knockdown of ApoL1 in Zebrafish Larvae Affects the Glomerular Filtration Barrier and the Expression of Nephrin. PLoS One. 2016; 11:e0153768. [PubMed: 27138898]

30. Zhaorigetu S, Wan G, Kaini R, et al. ApoL1, a BH3-only lipid-binding protein, induces autophagic cell death. Autophagy. 2008; 4:1079-82. [PubMed: 18927493]

31. Lan X, Jhaveri A, Cheng K, et al. APOL1 risk variants enhance podocyte necrosis through compromising lysosomal membrane permeability. Am J Physiol Renal Physiol. 2014; 307:F32636. [PubMed: 24899058]

32. Nichols B, Jog P, Lee JH, et al. Innate immunity pathways regulate the nephropathy gene Apolipoprotein L1. Kidney Int. 2014

33. Kopp JB, Nelson GW, Sampath K, et al. APOL1 genetic variants in focal segmental glomerulosclerosis and HIV-associated nephropathy. J Am Soc Nephrol. 2011; 22:2129-37. [PubMed: 21997394]

34. Sampson MG, Robertson CC, Martini S, et al. Integrative Genomics Identifies Novel Associations with APOL1 Risk Genotypes in Black NEPTUNE Subjects. J Am Soc Nephrol. 2015

35. Wan G, Zhaorigetu S, Liu Z, et al. Apolipoprotein L1, a novel Bcl-2 homology domain 3-only lipid-binding protein, induces autophagic cell death. J Biol Chem. 2008; 283:21540-9. [PubMed: 18505729]

36. Cheng D, Weckerle A, Yu Y, et al. Biogenesis and cytotoxicity of APOL1 renal risk variant proteins in hepatocytes and hepatoma cells. J Lipid Res. 2015; 56:1583-93. [PubMed: 26089538]

37. Khatua AK, Cheatham AM, Kruzel ED, et al. Exon 4 Encoded Sequence is a Major Determinant of Cytotoxicity of Apolipoprotein L1. Am J Physiol Cell Physiol. 2015 ajpcell.00384.2014.

38. Olabisi OA, Zhang JY, VerPlank L, et al. APOL1 kidney disease risk variants cause cytotoxicity by depleting cellular potassium and inducing stress-activated protein kinases. Proc Natl Acad Sci U S A. 2016; 113:830-7. [PubMed: 26699492]

39. Heneghan JF, Vandorpe DH, Shmukler BE, et al. BH3 domain-independent apolipoprotein L1 toxicity rescued by BCL2 prosurvival proteins. Am J Physiol Cell Physiol. 2015; 309:C332-47. [PubMed: 26108665]

40. Thomson R, Genovese G, Canon C, et al. Evolution of the primate trypanolytic factor APOL1. Proc Natl Acad Sci U S A. 2014; 111:E2130-9. [PubMed: 24808134]

41. Anderson BR, Howell DN, Soldano K, et al. In vivo Modeling Implicates APOL1 in Nephropathy: Evidence for Dominant Negative Effects and Epistasis under Anemic Stress. PLoS Genet. 2015; 11:e1005349. [PubMed: 26147622]

42. Vanhollebeke B, Pays E. The function of apolipoproteins L. Cell Mol Life Sci. 2006; 63:1937-44. [PubMed: 16847577]

43. Johnstone DB, Shegokar V, Nihalani D, et al. APOL1 null alleles from a rural village in India do not correlate with glomerulosclerosis. PLoS One. 2012; $7:$ :51546. [PubMed: 23300552]

44. Bruggeman LA, Wu Z, Luo L, et al. APOL1-G0 or APOL1-G2 Transgenic Models Develop Preeclampsia but Not Kidney Disease. J Am Soc Nephrol. 2016

45. Venkatareddy M, Wang S, Yang Y, et al. Estimating podocyte number and density using a single histologic section. J Am Soc Nephrol. 2014; 25:1118-29. [PubMed: 24357669]

46. Kikuchi M, Wickman L, Hodgin JB, et al. Podometrics as a Potential Clinical Tool for Glomerular Disease Management. Semin Nephrol. 2015; 35:245-55. [PubMed: 26215862]

47. Gutierrez MG, Saka HA, Chinen I, et al. Protective role of autophagy against Vibrio cholerae cytolysin, a pore-forming toxin from V. cholerae. Proc Natl Acad Sci U S A. 2007; 104:1829-34. [PubMed: 17267617]

48. Kloft N, Neukirch C, Bobkiewicz W, et al. Pro-autophagic signal induction by bacterial poreforming toxins. Med Microbiol Immunol. 2010; 199:299-309. [PubMed: 20454906]

49. Kloft N, Busch T, Neukirch C, et al. Pore-forming toxins activate MAPK p38 by causing loss of cellular potassium. Biochem Biophys Res Commun. 2009; 385:503-6. [PubMed: 19497299] 
50. Ma FY, Liu J, Nikolic-Paterson DJ. The role of stress-activated protein kinase signaling in renal pathophysiology. Braz J Med Biol Res. 2009; 42:29-37. [PubMed: 18982195]

51. He JC, Husain M, Sunamoto M, et al. Nef stimulates proliferation of glomerular podocytes through activation of Src-dependent Stat3 and MAPK1,2 pathways. J Clin Invest. 2004; 114:643-51. [PubMed: 15343382]

52. Feng X, Lu TC, Chuang PY, et al. Reduction of Stat3 activity attenuates HIV-induced kidney injury. J Am Soc Nephrol. 2009; 20:2138-46. [PubMed: 19608706]

53. Gu L, Dai Y, Xu J, et al. Deletion of podocyte STAT3 mitigates the entire spectrum of HIV-1associated nephropathy. Aids. 2013; 27:1091-8. [PubMed: 23343908]

54. Kasembeli AN, Duarte R, Ramsay M, et al. APOL1 Risk Variants Are Strongly Associated with HIV-Associated Nephropathy in Black South Africans. J Am Soc Nephrol. 2015

55. Bruggeman LA, Ross MD, Tanji N, et al. Renal epithelium is a previously unrecognized site of HIV-1 infection. J Am Soc Nephrol. 2000; 11:2079-87. [PubMed: 11053484]

56. Marras D, Bruggeman LA, Gao F, et al. Replication and compartmentalization of HIV-1 in kidney epithelium of patients with HIV-associated nephropathy. Nat Med. 2002; 8:522-6. [PubMed: 11984599]

57. Taylor HE, Khatua AK, Popik W. The innate immune factor apolipoprotein L1 restricts HIV-1 infection. J Virol. 2014; 88:592-603. [PubMed: 24173214]

58. McLaren PJ, Gawanbacht A, Pyndiah N, et al. Identification of potential HIV restriction factors by combining evolutionary genomic signatures with functional analyses. Retrovirology. 2015; 12:41. [PubMed: 25980612] 

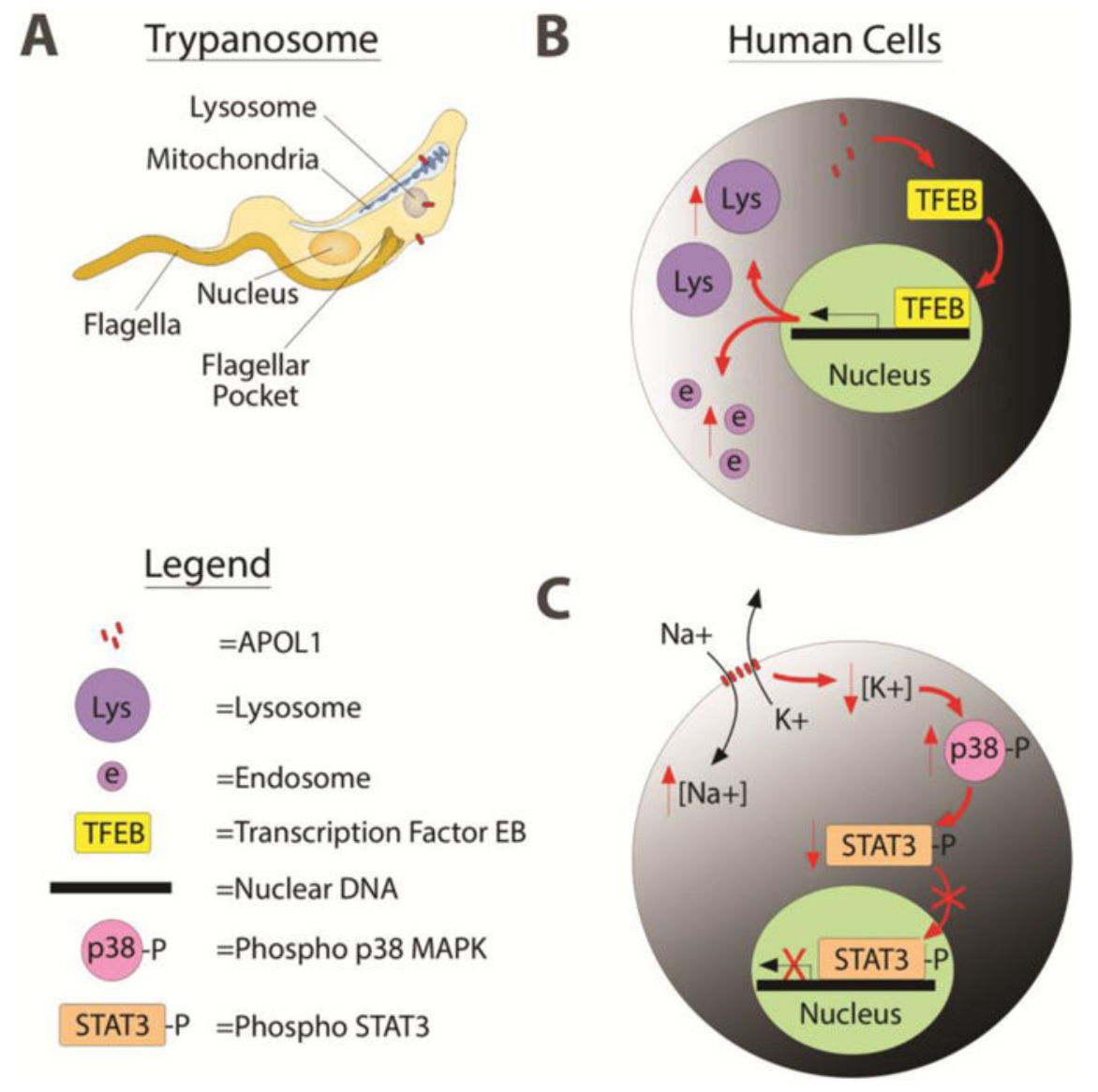

Figure 1. APOL1 localization and activities in the trypanosome has parallels in human cells (A) APOL1 enters the trypanosome as a component of HDL3 particles via endocytosis at the flagellar pocket and is trafficked to the lysosome. Independent studies report different mechanisms of trypanosomal killing. Activation of the APOL1 channel activity within the lysosome has been reported to cause lysosomal swelling and trypanolysis ${ }^{11}$. Others have found trypanolytic activity has localized to the plasma membrane ${ }^{13}$, or the mitochondria ${ }^{15}$. (B) Studies overexpressing APOL1 in human cells have reported that its expression leads to the movement of the transcription factor EB (TFEB) from the cytosol to the nucleus where it induces the transcription of genes necessary for the upregulation of lysosomal biogenesis and increases endosomal trafficking 57 . The upregulation of these pathways contributes to the restriction of HIV-1 infection in this report. (C) Other investigators found that overexpressed APOL1-G1 and-G2, but not G0, can localize to the plasma membrane where it allows the efflux of potassium driving the phosphorylation and activation of p38 MAPK and inhibition of gp130-STAT3 signaling that may change the cells transcriptional program ${ }^{38}$. Activation of the p38/STAT3 pathway downstream of channel activity at the plasma membrane was observed only after expression of the kidney disease risk variants of APOL1. 


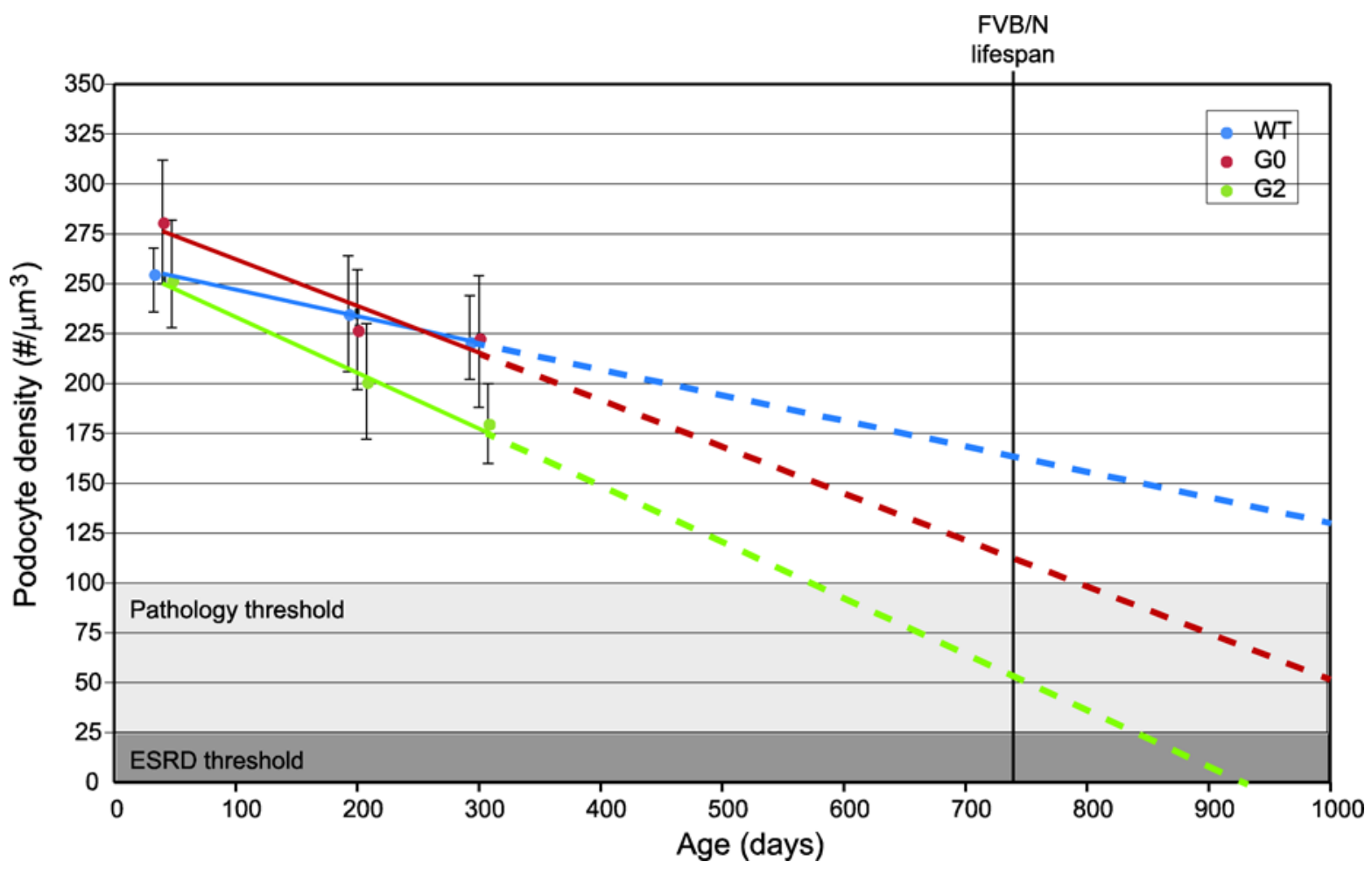

Figure 2. Trajectory of podocyte density change in FVB, Tg-G0 and Tg-G2 mice

Podocyte densities in wild-type, Tg-G0, and Tg-G2 mouse kidneys using a method developed by the Wiggins lab ${ }^{45}$. Data used to construct these trend lines were reported in Bruggeman et $\mathrm{al}^{44}$. In rodents and humans, once podocyte density decreases by $40 \%$, glomerular disease becomes progressive ${ }^{46}$. At 40d, podocyte densities were similar in FVB, Tg-G0 and Tg-G2 mice. Subsequently, podocyte density in the Tg-G2 mice were significantly lower but remained above the threshold for overt kidney disease through 300 days of age. We extrapolated the rate podocyte density change from the empirical data and found Tg-G2 mice would not be at risk for overt kidney disease within the normal lifespan of FVB/N mice, which is consistent with the observation that individuals with APOL1 risk genotypes are not destined to develop kidney disease in the absence of a "second hit" (e.g. HIV infection. 